\title{
New insights on the accuracy of photometric redshift measurements
}

\author{
M. Massarotti ${ }^{1,2}$, A. Iovino ${ }^{2}$, A. Buzzoni ${ }^{2,3}$, and D. Valls-Gabaud ${ }^{4}$ \\ 1 Osservatorio Astronomico di Capodimonte, Via Moiariello 16, 80131 Napoli, Italy \\ 2 Osservatorio Astronomico di Brera, Via Brera 28, 20121 Milano, Italy \\ e-mail: iovino@brera.mi.astro.it \\ 3 Telescopio Nazionale Galileo, PO Box 565, 38700 Santa Cruz de La Palma (TF), Spain \\ e-mail: buzzoni@tng.iac.es \\ 4 CNRS UMR 5572, Observatoire Midi-Pyrénées, 14 Av. E. Belin, 31400 Toulouse, France \\ e-mail: dvg@ast.obs-mip.fr
}

Received 2 July 2001 / Accepted 4 October 2001

\begin{abstract}
We use the deepest and most complete redshift catalog currently available (the Hubble Deep Field (HDF) North supplemented by new HDF South redshift data) to minimize residuals between photometric and spectroscopic redshift estimates. The good agreement at $z_{\text {spec }}<1.5$ shows that model libraries provide a good description of the galaxy population. At $z_{\mathrm{spec}} \geq 2.0$, the systematic shift between photometric and spectroscopic redshifts decreases when the modeling of the absorption by the interstellar and intergalactic media is refined. As a result, in the entire redshift range $z \in[0,6]$, residuals between photometric and spectroscopic redshifts are roughly halved. For objects fainter than the spectroscopic limit, the main source of uncertainty in photometric redshifts is related to photometric errors, and can be assessed with Monte Carlo simulations.
\end{abstract}

Key words. galaxies: distances and redshifts, evolution, ISM, intergalactic medium - methods: data analysis techniques: photometric

\section{Introduction}

Reliable spectroscopy of galaxies fainter than $I_{\mathrm{AB}} \sim 24$ is still an unattainable goal even with new-generation telescopes. To estimate the redshift for a majority of these objects one must therefore rely on multicolor observations in order to reconstruct, at very low resolution, the apparent spectral energy distribution (SED).

Much efforts have been devoted, in the recent literature, to improve the accuracy of the photometric approach compared with direct spectroscopy. Photometric redshift techniques have been extensively applied, in particular, to the ultra-deep images of the Hubble Deep Field North (HDFN, Williams et al. 1996; see also Ferguson et al. 2000) to study the luminosity function, the evolution of the star formation rate (SFR) density, and the clustering properties of the galaxies observed there (Madau et al. 1996; Connolly et al. 1997; Sawicki et al. 1997; Franceschini et al. 1998; Pascarelle et al. 1998; Connolly et al. 1998; Miralles \& Pelló 1998; Roukema et al. 1999; Arnouts et al. 1999).

The sample of relatively bright HDFN objects with reliable spectroscopic redshifts is steadily increasing and now amounts to about 150 objects, a value that nearly

Send offprint requests to: M. Massarotti, e-mail: massarot@brera.mi.astro.it doubles the initial list of Sawicki et al. (1997). About $20 \%$ of these objects are beyond $z \geq 2.0$, thus allowing a more robust statistical comparison between spectroscopic and photometric estimates of $z$ even for the most distant objects in the field.

We previously discussed (Massarotti et al. 2001a, Paper I hereafter) the performance of the SED fitting method as a function of the template set adopted to reproduce galaxy colors. Our results suggested that, as long as both normal and starburst galaxies are taken into account in the reference templates, the photometric redshift at $z<1.5$ is constrained by a reliable detection of the $4000 \AA$ Balmer break in the target SED. At larger redshifts, on the contrary, the main constraint is set by modeling the effects of the interstellar (ISM) and intergalactic (IGM) absorption, which modulate the SEDs at different wavelengths. In this paper, we further discuss some important aspects of this problem, and suggest an improved model for the ISM and IGM absorption which sensibly increases the accuracy of the method at $z \gtrsim 2$.

Photometric redshifts are estimated by comparing the observed broad-band colors of galaxies with the library of model fluxes provided by the code of Buzzoni (1989, 1995, 2001; hereafter BUZ). Results obtained with the codes of Bruzual \& Charlot (1993, BC) and 
Fioc \& Rocca-Volmerange (1997, FRV) are also reported, since these theoretical models have also been extensively used in the literature.

The key steps of our procedure are summarized in Sect. 2, where we also introduce the model libraries. The redshift database used in our analysis is presented in Sect. 3, comparing the $z_{\text {spec }}$ distribution with our $z_{\text {phot }}$ output. The ISM and IGM effects are more specifically dealt with in Sect. 4, while in Sect. 5 we discuss the match between models and observations even at the faintest magnitude limit of the HDFN data. Section 6 summarizes our conclusions.

\section{Input ingredients and fitting algorithm}

As in Paper I, the photometric catalog of Fernández-Soto et al. (1999) will be used as a reference in our analysis. It is made up by 1067 objects identified from the HST WFPC2 images in the four photometric bands, namely $U_{300}, B_{450}$, $V_{606}$, and $I_{814}$, and completed with infrared observations in the Johnson $J, H$, and $K$ bands with the IRIM camera at KPNO (Dickinson 1998).

Galaxy model libraries have been assembled following the strategy of Paper I. A notable change in the present calculations involves a slight enlargement of the set of starburst models in order to cover a wider range of physical parameters and ensure a more accurate description of the colors of the most distant galaxies.

To the Leitherer et al. (1999) starburst sequence of Paper I, namely for $t=50,100$, 500, and $800 \mathrm{Myr}$, with $Z_{\odot}$ and Salpeter IMF, we added here two supplementary models at $t=10$ and $250 \mathrm{Myr}$, and included evolution for $Z_{\odot} / 20$ metallicity in addition to the solar case previously considered.

In order to account for recent star formation superimposed on quiescent red (elliptical) galaxies, we also considered a new set of templates. These are obtained adding the 50 Myr starburst spectrum (with Salpeter IMF and $Z_{\odot}$ ) to simple stellar population models for a multi-component fit to the galaxy SED. In this way the overall color distribution of early type galaxies at $z<1.5$, as found by Franceschini et al. (1998) and Rodighiero et al. (2001), is much better accounted for.

As a first step, the ISM and IGM absorptions have been modeled in as in Paper I. Galaxy model fluxes have been corrected for ISM effects following Calzetti (1999). As in Paper I, the $E(B-V)$ color excess has been set as a free fitting parameter which could take the discrete values $0.0,0.05,0.1,0.2,0.3,0.4$. The IGM absorption as a function of redshift follows the analaysis by Madau (1995).

According to our SED fitting method, we searched for the "best template" solution as which minimizes the general $\chi^{2}$ function (see Eq. (1) of Paper I). In the multi-component fit to the galaxy SED the $\chi^{2}$ functional takes the form:

$$
\chi^{2}=\sum_{i=1}^{N} \frac{\left[f_{i}^{\text {obs }}-s_{\mathrm{ssp}} f_{i, \mathrm{ssp}}^{\text {temp }}(z)-s_{\text {burst }} f_{i, \text { burst }}^{\text {temp }}(z)\right]^{2}}{\sigma_{i}^{2}},
$$

where $f_{i, \text { ssp }}^{\text {temp }}$ and $f_{i \text {, burst }}^{\text {temp }}$ are the template fluxes of the quiescent galaxy model and of the burst model, respectively, scaled by $s_{\text {ssp }}$ and $s_{\text {burst }}$, and $N$ is the number of filters.

For galaxies with $z_{\text {spec }}$ available $\left(N_{\mathrm{g}}\right)$ we define two simple statistical moments:

$\overline{\Delta z}=\sum^{N_{\mathrm{g}}} \frac{\left(z_{\mathrm{spec}}-z_{\mathrm{phot}}\right)}{N_{\mathrm{g}}}$,

and

$\sigma_{z}^{2}=\sum^{N_{\mathrm{g}}} \frac{\left[\left(z_{\mathrm{spec}}-z_{\mathrm{phot}}\right)-\overline{\Delta z}\right]^{2}}{N_{\mathrm{g}}-1}$,

to study residuals between photometric and spectroscopic redshift values.

Catastrophic outliers (that is, galaxies with $\mid z_{\mathrm{spec}}-$ $\left.z_{\text {phot }} \mid>1.0\right)$ are clipped from the galaxy distribution to compute representative values for $\overline{\Delta z}$ and $\sigma_{z}^{2}$.

\section{The spectroscopic sample}

Cohen et al. (2000) provided an exhaustive compilation of all spectroscopic redshifts measured with the Keck telescope in the HDFN proper and flanking fields. The work includes previously published data, new redshifts measurements, and corrections to erroneous values given in the literature. This list represents the deepest and most complete redshift catalog for the HDFN to date.

Fernández-Soto et al. (2001) matched photometric and redshift catalogs in the HDFN proper. They crosscorrelated coordinates and magnitudes, resulting in a list of 146 sources (see Table 2 therein). We adopt here their naming convention and follow their considerations regarding some galaxies with discordant $z_{\mathrm{spec}}$ vs. $z_{\text {phot }}$ values, except for the following few objects.

HDF36441-1410: we assume that the spectroscopic measure $z_{\text {spec }}=2.267$ is correct. Fernández-Soto et al. (2001) claimed that features identifications in the spectrum are compatible with their estimated $z_{\text {phot }}=0.01$, but HDF36441-1410 is a $U$ drop-out star-forming galaxy $\left(U_{300}-B_{450} \sim 1.7, V_{606}-I_{814} \sim 0.0\right)$. These colors (blue in $V_{606}-I_{814}$, red in $\left.U_{300}-B_{450}\right)$ can be explained only as consequence of the IGM absorption of UV photons in the $U_{300}$ band, and are incompatible with $z \sim 0$ starburst colors.

HDF36446-1227: we prefer the spectroscopic value $z_{\text {spec }}=2.035$ proposed by Lowenthal et al. (1997), to the (revised) value $z_{\text {spec }}=2.500$ reported by Cohen et al. (2000) and accepted by Fernández-Soto et al. (2001). In the redshift range $z_{\text {spec }} \in[2,2.3]$ there is no galaxy bluer than HDF36446-1227 in $U_{300}-B_{450}$, and according to 

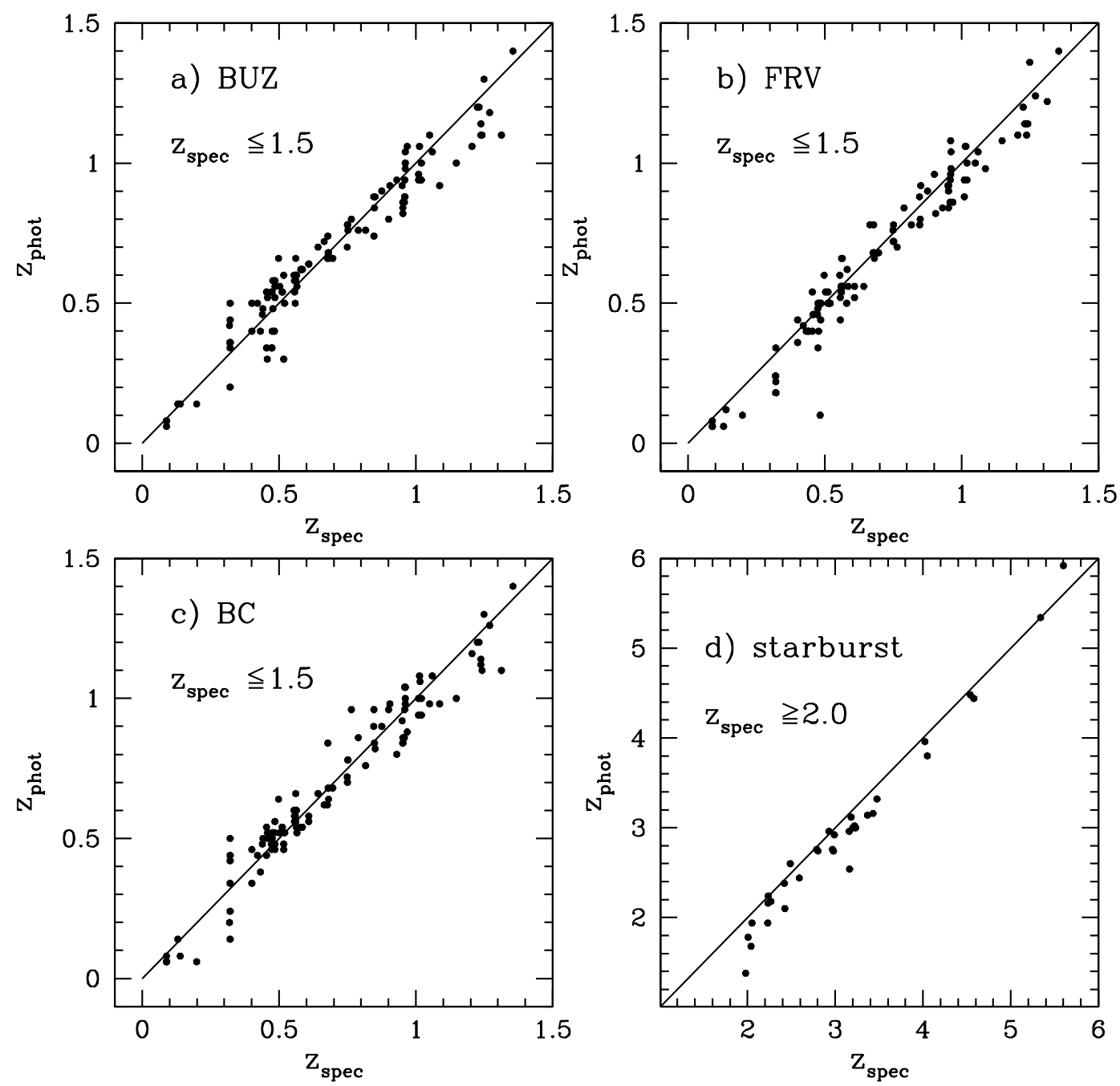

Fig. 1. Comparison between spectroscopic and photometric redshifts according to different template libraries (see the text for acronyms). The solid line is for $\Delta z=0.0$. Note the different scale for panel $\mathbf{d}$ ).

the starburst templates of our models its optical colors are incompatible with $z_{\text {spec }}>2.3$ (see Sect. 4.2).

HDF36396-1230, 36494-1317, and 36561-1330: for these three galaxies we follow Cohen (2001), who adopts the redshift values given by Fernández-Soto et al. (2001).

HDF36478-1256: with $z_{\text {spec }}=2.931$, this galaxy was tagged as a catastrophic outlier in all previous works (see e.g. Fernández-Soto et al. 1999; Bolzonella et al. 2000; Fernández-Soto et al. 2001; Fontana et al. 2000). Arnouts et al. (1999) suggest that the photometry could be incorrect because of the complex source morphology. Given the unclear situation and the large discrepancy for this galaxy (our photometric redshift estimate is $z_{\text {phot }}=0.26$ ) we excluded it from the catalog.

Six new galaxies from the HDF-South (HDFS) have also been added to our list. Three of them are from Glazebrook et al. (2001) with $z_{\text {spec }}=0.580,0.582$, and 0.696 , and three are from Cristiani et al. (2000) with $z_{\text {spec }}=0.565,2.79$, and 3.2 respectively. The photometry was obtained from the web page of the Stony Brook group (see http://www.ess.sunysb.edu/astro/hdfs) in the four WFPC2 filters, and in the $J, H$, and $K$ infrared bands by the SOFI camera at NTT (Da Costa et al. 2001).

Our final spectroscopic catalog contains 146 objects, 34 of them with $z_{\text {spec }} \geq 2$.

\subsection{Comparison between $z_{\text {spec }}$ and $z_{\text {phot }}$}

In Fig. 1 we compare spectroscopic and photometric redshifts obtained with different sets of reference galaxy models.

At $z_{\text {spec }}<1.5$ (panels $a, b, c$ ) the agreement is excellent, regardless of the template set chosen. Dispersion values of the photometric redshift estimates are $\sigma_{z}=0.078$, 0.074, 0.072 for the BUZ, FRV and BC libraries, respectively, while systematic errors are $\overline{\Delta z}=0.007,0.026$, 0.004 . There are no galaxies with $\left|z_{\text {spec }}-z_{\text {phot }}\right|>0.5$.

Since in this redshift range the accuracy of the method depends mainly on the ability of models to reproduce the $4000 \AA$ Balmer break along the whole Hubble morphological sequence (including starburst galaxies), one can 
conclude that all model libraries contain enough information to match properly the SEDs.

For the same sample, at $z_{\text {spec }}<1.5$, Fontana and collaborators obtained $\sigma_{z}=0.084$ and $\overline{\Delta z}=0.033$ (see the web page http://www.mporzio.astro.it/HIGHZ/ HDF.html). Fernández-Soto et al. (1999) obtained $\sigma_{z}=$ 0.116 and $\overline{\Delta z}=-0.007$, further improved to $\sigma_{z}=0.107$ and $\overline{\Delta z}=-0.004$ after including starburst galaxies in their template library (Fernández-Soto et al. 2001). Bolzonella et al. (2000) obtained $\sigma_{z} \sim 0.082$, using a smaller spectroscopic sample, that did not account for the new spectroscopic redshifts from Cohen and collaborators.

The situation becomes more complex at $z_{\text {spec }} \geq 2$ (see panel d of Fig. 1). The dispersion increases to $\sigma_{z}=0.177$ and there are two objects with $\left|z_{\text {spec }}-z_{\text {phot }}\right|>0.5$. In this redshift range almost all galaxies are best fit by starburst models, and we therefore focussed on the Leitherer et al. (1999) templates.

Part of the larger scatter can certainly result from the fainter magnitudes (lower signal-to-noise ratio), but a systematic shift $(\overline{\Delta z}=0.156)$ is significant in panel $\mathrm{d}$, and cannot be explained in terms of photometric errors only. The tendency to underestimate redshifts for $z_{\text {spec }} \geq 2$ seems to be a common feature of all photometric studies found in the literature, regardless of the specific choice of theoretical and/or empirical template library.

The only exception in this regard are the results of Fernández-Soto et al. (2001) which show systematics in the opposite sense, with $\overline{\Delta z}=-0.136$. These authors did not however take into account explicitly the ISM reddening modulation of the template SEDs (the average amount of dust that the empirical templates do contain may not be entirely representative of the dust content of galaxies at high redshifts). As we noted in Paper I, this causes a significant overestimate (by about $10-20 \%$ ) of $z$ at large distances, because the IGM absorption, which increases with redshift, is forced to mimic color reddening.

The presence of dust in starburst galaxies at high redshift has been confirmed by analyzing galaxy colors with the SED fitting method (see Massarotti et al. 2001b, and references therein). Meurer et al. (1999) adopted an entirely empirical approach based on the correlation between color reddening, dust absorption and far infrared flux for local starburst galaxies (supposed to be also valid at high redshifts). According to their results (see also Meurer et al. 1997; Sawicki \& Yee 1998), at $\langle z\rangle=2.75$ a color-luminosity correlation is already in place: more luminous galaxies (i.e. galaxies with available spectroscopic redshifts) have a larger dust content.

The observed systematic shift in the $z_{\text {spec }}$ vs. $z_{\text {phot }}$ distribution of Fig. 1, calls therefore for a revised analysis of the ISM and IGM effects in the $z \gtrsim 2$ data.

\section{A revised approach to IGM and ISM absorption}

In Paper I we showed that at high redshifts the SED fitting method provides stable estimates of galaxy redshifts, even when using an extremely poor template library, while the critical ingredients are dust reddening and IGM attenuation. It is important to explore in some detail the physical processes of photon absorption in both cases.

\subsection{The IGM opacity as a function of redshift}

Following Madau (1995), the Ly $\alpha$ forest contribution to the attenuation of the SED caused by the IGM absorption can be written in terms of an effective optical depth as

$\frac{F_{\text {obs }}(\lambda, z)}{F_{\text {intr }}(\lambda)}=\mathrm{e}^{-\tau_{\text {eff }}}$,

with

$\tau_{\text {eff }}=A \times\left(\lambda_{\text {obs }} / \lambda_{\alpha}\right)^{1+\gamma}$,

where $F_{\text {obs }}(\lambda, z)$ and $F_{\text {intr }}(\lambda)$ are the observed and intrinsic fluxes respectively, $\lambda_{\text {obs }}=\lambda \times(1+z)$, and $\lambda_{\alpha}=1216 \AA$.

Press et al. (1993), in their analysis of the distribution of Ly $\alpha$ forest lines in a sample of 29 quasars at $z \in[3.1,4.5]$, found $(A, \gamma)=(0.0036,2.46)$, which are the values usually adopted in the literature.

In a recent paper, Scott et al. (2000) studied a sample containing 97 quasars at $z \in[1.7,3.6]$, a redshift range complementary to that studied by Press et al. (1993). Considering absorption lines above the threshold $W>$ $0.16 \AA$, they obtain $(A, \gamma)=(0.00759,1.35)$. The IGM transmission at $z<3$ is therefore larger than the one expected by extrapolating the Press et al. (1993) results at these redshifts.

We decided to adopt the Scott et al. (2000) results to describe the IGM absorption at $z<3$ and the Press et al. (1993) results at $z>4$. In the intermediate redshift range (i.e. between $3<z<4$ ), the $A$ and $\gamma$ coefficients of Eq. (5) have been interpolated linearly imposing as boundary limits $(A, \gamma)=(0.00759,1.35)$ at $z=3$ and $(A, \gamma)=(0.0036,2.46)$ at $z=4$.

A useful way to match our adopted theoretical scheme with the observations is to derive from Eq. (5) the expected IGM flux attenuation $\left(D_{\mathrm{A}}\right)$ in the spectral range $\lambda \in[1050,1170] \AA$ as a function of redshift. This can be directly compared with the observed deficit in quasars fluxes due to the accumulated absorption of the Ly $\alpha$ lines in the IGM. By definition,

$D_{\mathrm{A}}(z)=1-\frac{\int_{1050}^{1170} F_{\mathrm{obs}}(\lambda, z) \mathrm{d} \lambda}{\int_{1050}^{1170} F_{\mathrm{intr}}(\lambda) \mathrm{d} \lambda}$.

Our results are summarized in Fig. 2, which collects observations from different sources. The data cannot be described by a single power law, since the slope of $D_{\mathrm{A}}(z)$ changes in the interval $z \in[3,4]$. The Press et al. (1993) solution (adopted by Madau 1995) sensibly overestimates the IGM opacity at $z<3.5$; the opposite happens with the Scott et al. (2000) results when extending them beyond $z>3.5$. Note, on the contrary, that our simple parameterization fits nicely the $D_{\mathrm{A}}(z)$ evolution over the entire range of redshift, from $z=2$ to $z=6$. 


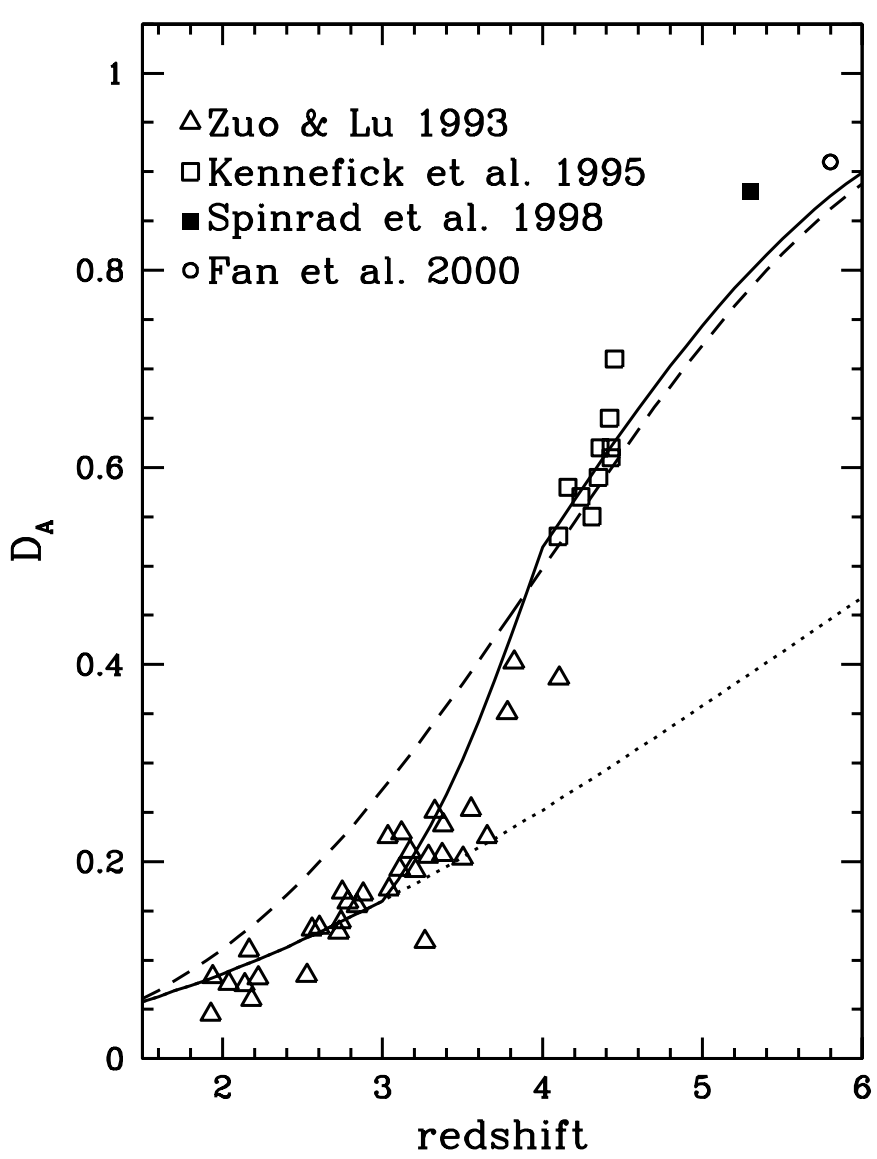

Fig. 2. The deficit $D_{\mathrm{A}}$ in quasars fluxes due to the accumulated absorption of the Ly $\alpha$ forest. The dashed line is obtained by describing the IGM opacity according to the prescriptions by Madau (1995), while the dotted line are those by Scott et al. (2000). The solid line is the model adopted in this paper. References for the data points are indicated. The small difference between the Madau values of $D_{\mathrm{A}}$ and ours at $z>4$ is due to the contribution of Ly limit systems (Madau 1995, Eq. (13)), which was not included in Madau's formulation.

We repeated the photometric redshift estimate for the $z_{\text {spec }} \geq 2.0$ objects adopting our new prescriptions for the IGM absorption. For $z>2.5$, only one outlier (namely HDF36512-1349 at $z_{\text {spec }}=3.162$ ) is found at $\left|z_{\text {spec }}-z_{\text {phot }}\right|>0.5$. In the Cohen et al. (2000) list the spectroscopic redshift of this object is reported as (very) uncertain.

Excluding this source, the dispersion decreases to $\sigma_{z}=$ 0.129 (cf. Fig. 3c). More importantly, the tendency to underestimate galaxy redshift almost disappears $(\overline{\Delta z}=$ 0.042 at $z_{\text {spec }} \geq 2.5$ ). This is not true however in the range $z \in[2,2.5]$, where the results are still problematic.

\subsection{The ISM opacity and the bump at $2175 \AA$}

In the redshift range $z_{\text {spec }} \in[2,2.5]$, the $912 \AA$ Lyman break has not yet entered the $U_{300}$ band and therefore galaxies can still be detected in this filter, although with a major dimming effect by the IGM absorption.
The $U_{300}$ magnitude does not effectively constrain $z$ in the SED fitting method, because of its poor signal-to-noise ratio $^{1}$. The $\chi^{2}$ merit function is mainly modulated in this redshift range by the optical information coming from the $B_{450}, V_{606}$ and $I_{814}$ bands. It is therefore plausible that any bias in the $z$ estimate is linked in some way with the $B_{450}-V_{606}$ and $V_{606}-I_{814}$ colors.

At $z \sim 2$ the $B_{450}$ band is probing the rest-frame emission at about $1500 \pm 300 \AA$, while the $V_{606}$ band samples the wavelength range $2000 \pm 300 \AA$, and the $I_{814}$ band that at $2700 \pm 300 \AA$. Between 1500 and $2700 \AA$, the Leitherer et al. (1999) starburst models indicate that the galaxy spectral slope steepens with increasing wavelength so that, in general, $\left(B_{450}-V_{606}\right)>\left(V_{606}-I_{814}\right)$ in the $\mathrm{AB}$ mag scale.

This is confirmed by Fig. 4, where the color diagram for the 100 Myr starburst model with Salpeter IMF and $Z_{\odot}$ is shown. Dust reddening can only strengthen this effect: dust absorption reddens $B_{450}-V_{606}$ more than $V_{606}-I_{814}$ (see in Fig. 4 the reddening vectors). Moreover, at $z>2.5$ the Lyman series enters the $B_{450}$ band and the $B_{450}-V_{606}$ color is further reddened by the IGM absorption.

In Fig. 4 we also plotted the HDFN $U$ drop-out sample (triangles) selected according to the prescriptions of Meurer et al. (1999) $\left(U_{300}-B_{450} \geq 1.3, V_{606}-I_{814}<0.5\right.$, and $\left.B_{450} \leq 26.8\right)$. A large fraction $(24 \%)$ of these galaxies displays observed colors $\left(B_{450}-V_{606}\right)<\left(V_{606}-I_{814}\right)$ and 3 out of the 11 sources at $z_{\text {spec }} \in[2,2.5]$ are among these. Note that for galaxies with spectroscopic reddshifts, the color errors are $\leq 0.02$ mags, so it is difficult to explain such large deviations from models in terms of photometric errors only. To account in a consistent way for the deviating $B_{450}-V_{606}$ and $V_{606}-I_{814}$ colors, one could think that a dimming in the $V_{606}$ flux is intervening in the data, at about the rest-frame wavelength corresponding to the $2175 \AA$ bump of the dust extinction law in the Milky Way (Seaton 1979) or the Large Magellanic Cloud (Fitzpatrick 1986).

We tried to superimpose the bump $C(\lambda)$ on Calzetti's law, in the form of a Drude profile according to Fitzpatrick \& Massa (1988, see also Calzetti et al. 1994):

$C(\lambda)=\alpha \times \tau_{\mathrm{B}}^{1} \frac{x^{2}}{\left(x^{2}-x_{0}^{2}\right)^{2}+\gamma^{2} x^{2}}$,

where $x=\lambda^{-1}\left(\mu \mathrm{m}^{-1}\right), x_{0}=\lambda_{0}^{-1}=4.6 \mu \mathrm{m}^{-1}$ is the central wavelength of the dust bump, $\gamma=0.95 \mu \mathrm{m}^{-1}$ is its $F W H M$, and $\tau_{\mathrm{B}}^{\mathrm{l}}=E(B-V) /(0.935 \times 0.44)$ is the Balmer optical depth, as inferred from the difference in the optical depth between the nebular emission lines $\mathrm{H} \alpha$ and $\mathrm{H} \beta$ (see Calzetti et al. 1994; Calzetti 2000). Introducing the bump, the differential dust attenuation curve takes the form $A(\lambda)=[k(\lambda)+C(\lambda)] \times E(B-V)$, where $k(\lambda)$ follows Calzetti's law, and $C(\lambda)$ comes from Eq. (7).

${ }^{1}$ Note that, paradoxically, the same spectral signature which defines so effectively the $U$ drop-out sample at $z \geq 2$ causes the $U_{300}$ information to be rather useless in the SED fitting method. 


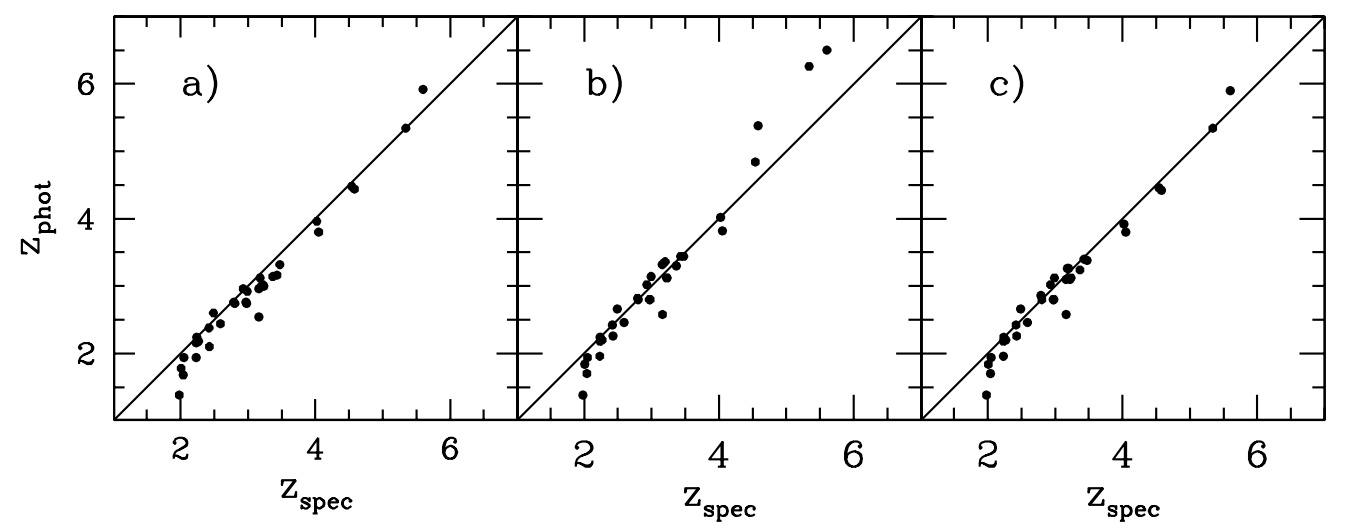

Fig. 3. Comparison between spectroscopic and photometric redshifts at $z_{\text {spec }} \geq 2$. Panel a) the IGM opacity follows the prescription by Madau (1995); panel b) by Scott et al. (2000); panel c) the formulation discussed in this paper. The solid line is for $\Delta z=0.0$. It should be noted that when the Scott et al. (2000) results are adopted, the IGM opacity is underestimated at $z>3.5$, and, as a consequence, photometric redshifts overestimate the spectroscopic ones.

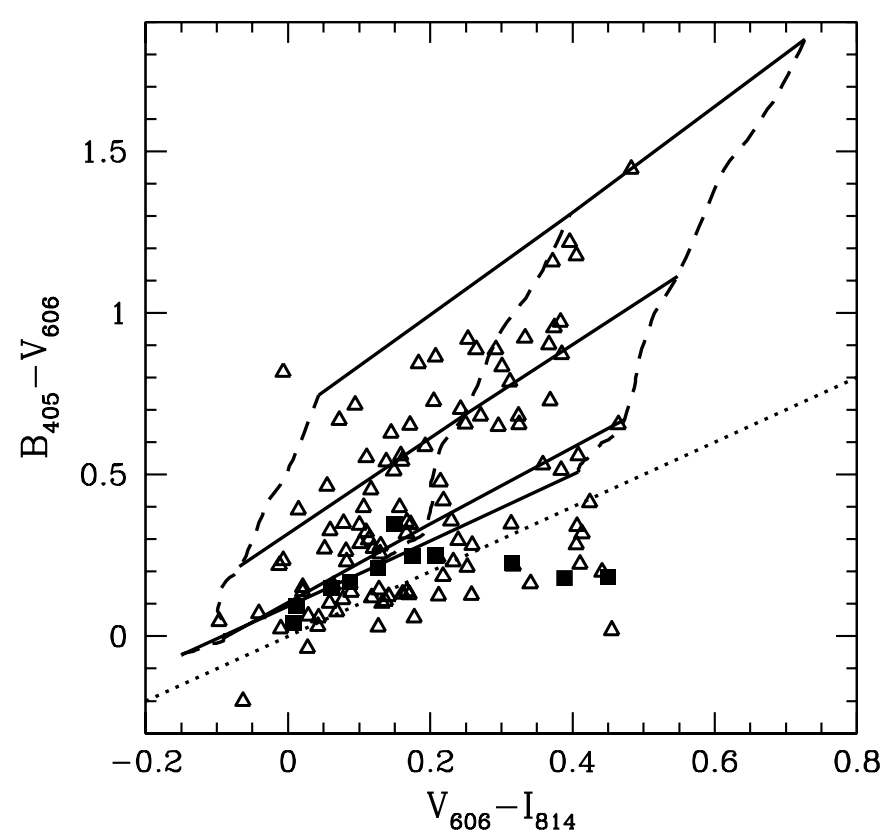

Fig. 4. Observed colors of $U$ drop-out galaxies (triangles), galaxies with spectroscopic redshifts in the range $z \in[2,2.5]$ (filled squares), and model colors of starburst galaxies in the redshift range $z \in[2,3.5]$ (see the text for details). Dashed lines show the color evolution of templates for increasing redshift (from bottom to top) at $E(B-V)=0.0,0.2,0.4$ (from left to right). Solid lines define reddening vectors at $z=2.0,2.5,3.0,3.5$ (from bottom to top), that is, the loci of templates at fixed redshift with variable amounts of dust. The dotted line corresponds to $B_{450}-V_{606}=V_{606}-I_{814}$.

In Fig. 5, the differential dust attenuation curve is shown (for $E(B-V)=0.2$ ) without the bump (solid line) and with a bump of intrinsic depth $\alpha=0.25$ (dashed line). For comparison the Milky Way extinction curve is also shown (Seaton 1979), where the intrinsic depth of the bump is $\alpha \sim 0.8$ ( $\alpha \sim 0.4$ for the Large Magellanic Cloud, see for example Calzetti et al. 1994, Fig. 13).

When the $2175 \AA$ bump is superimposed on the dust attenuation law, the locus occupied by starburst galax-

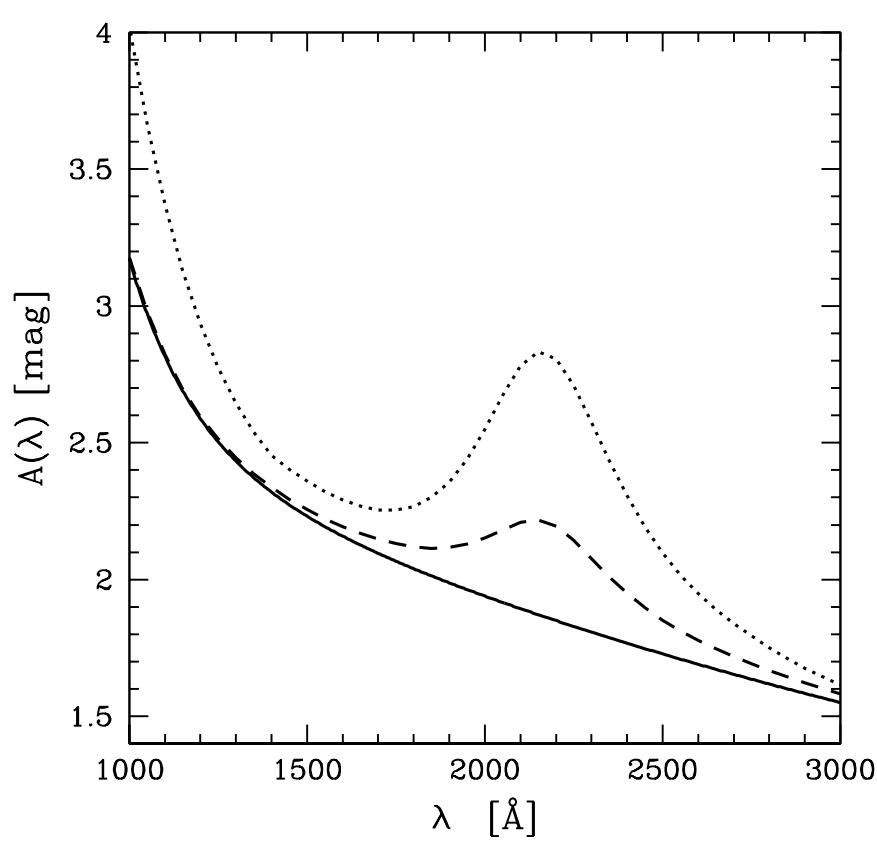

Fig. 5. Differential dust absorption (for $E(B-V)=0.2$ ) according to Calzetti's law (solid line), a superposition of Calzetti's law with a $2175 \AA$ bump of amplitude $\alpha=0.25$ (dashed line), and the Seaton (1979) extinction curve for the Milky Way (dotted line).

ies in the $\left(B_{450}-V_{606}\right)$ vs. $\left(V_{606}-I_{814}\right)$ plane (in the range $z \in[2,2.5])$ broadens to include galaxy colors with $B_{450}-V_{606}<V_{606}-I_{814}$ (see Fig. 6). Adding the $2175 \AA$ bump ( $\alpha=0.1,0.25)$ enables us to better reproduce galaxy colors in the range $z_{\text {spec }} \in[2,2.5]$ and significantly improves the agreement between photometric and spectroscopic redshift estimates. While $\sigma_{z}=0.206, \overline{\Delta z}=0.167$ (without the bump), the values decrease when the bump is allowed to $\sigma_{z}=0.094, \overline{\Delta z}=0.060$. For the three galaxies (see Fig. 6) which adopt a $2175 \AA$ bump value different from zero in their fit, the $\chi^{2}$ value is, on the mean, 4 times smaller than the one obtained with no bump, confirming the statistical significance of our improvement. 


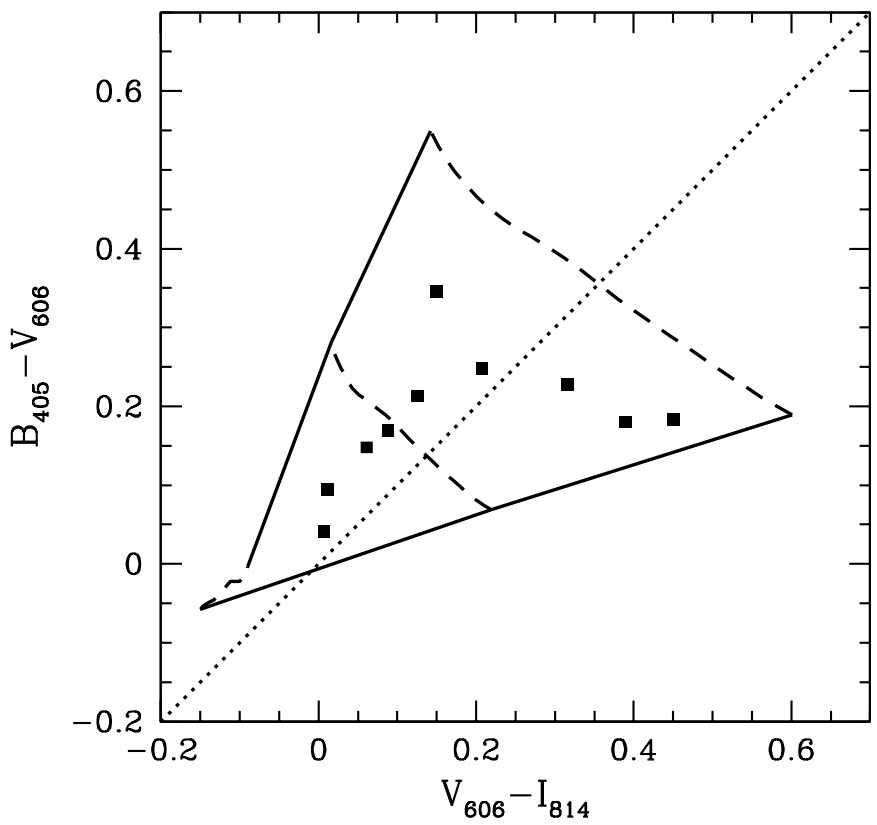

Fig. 6. Observed colors of spectroscopic galaxies (squares) and model colors of starburst galaxies in the redshift range $z \in[2,2.5]$. Dashed lines and solid lines have the same meaning as in Fig. 4, but in this case reddening in colors due to dust absorption is obtained superimposing on Calzetti's law a $2175 \AA$ bump with amplitude $\alpha=0.25$. Again, the dotted line corresponds to $B_{450}-V_{606}=V_{606}-I_{814}$. As consequence of the rotation of the reddening vectors, model colors are able to describe galaxy colors with $\left(B_{450}-V_{606}\right)<\left(V_{606}-I_{814}\right)$.

The question of the presence of the $2175 \AA$ bump in the dust attenuation law is still hotly debated in the literature. Gordon et al. (1997) claimed that radiative transfer effects associated with the spatial distribution of dust cannot (alone) decrease the feature below observational detectability. Therefore, the lack of a $2175 \AA$ bump in a starburst galaxy must be explained as a consequence of physical and chemical properties of dust grain. Reversing the argument, in a starburst with LMC-like dust, geometrical effects alone would certainly not prevent its possible detection.

In contrast, Granato et al. (2000) found, with their dust model for starbursts, that the $2175 \AA$ bump is absent due to purely geometrical effects. In the far-UV, the global attenuation in starburst models is dominated by the component associated with molecular clouds, where stars are born, and from which they gradually escape. Clouds have such large optical depths that the ultraviolet light from the stars inside is completely absorbed, and the shape of the attenuation curve does not contain information on the UV-optical properties of grains.

On the other hand, relying on the same theoretical framework, the results of Silva et al. (1998) lead us to conclude that virtually all the stars embedded in dark molecular clouds escape the regions of harder absorption in less than $10^{8}$ yr (cf. also Buzzoni 2001 on this subject). In such cases, the cirrus component dominates and a (weak) $2175 \AA$ bump should eventually appear in the dust attenuation law.

Calzetti et al. (1994) did explicitly avoid the $2175 \AA$ bump wavelength region when calibrating their starburst attenuation law, as the possible presence of absorption bump would destroy a linear correlation between the UV power law slope $\beta$ and $\tau_{\mathrm{B}}^{\mathrm{l}}$. When fitting a posteriori the residuals between observed spectra and the appropriate linear law $(\log F(\lambda)=\beta \log \lambda+$ const.) according to the Fitzpatrick \& Massa (1988) parameterization, they obtained a mean value $\alpha=0.07$ for the $2175 \AA$ bump amplitude. It should be noted, though, that among the objects in their sample with $\tau_{\mathrm{B}}^{\mathrm{l}} \geq 0.25(E(B-V) \geq 0.1$, see again Calzetti et al. 1994, Fig. 13) - that is, those where the presence of the feature is easier to detect - about $35 \%$ are compatible with $\alpha=0.25$.

Our results support the presence of the $2175 \AA$ bump in some galaxies observed in the spectroscopic sample: values of $\alpha$ up to 0.25 allow a better description of galaxy colors and redshifts. Higher-quality spectra for these controversial objects are needed to assess beyond any reasonable doubt the actual presence of the bump.

\section{Updating our results}

The inclusion of the refinements discussed above in the SED model libraries significantly improved the agreement between photometric and spectroscopic redshifts at $z_{\text {spec }} \geq 2.0$. The dispersion decreases to $\sigma_{z}=0.117$, while the systematic error is $\overline{\Delta z}=0.048$ (see Fig. 7), with no catastrophic outliers.

Using the same sample in the redshift range $z_{\text {spec }} \geq$ 2.0, Fontana and collaborators obtained $\sigma_{z}=0.232$, $\overline{\Delta z}=0.144$ (with one catastrophic outlier), FernándezSoto et al. (1999) obtained $\sigma_{z}=0.299, \overline{\Delta z}=0.186$ (with two catastrophic outliers), while for Fernández-Soto et al. (2001) $\sigma_{z}=0.234$ and $\overline{\Delta z}=-0.136$ (with one catastrophic outlier). With a smaller spectroscopic sample, Bolzonella et al. (2000) estimated $\sigma_{z} \sim 0.36$ (with one catastrophic outlier).

A further small improvement can be obtained by taking into account dust opacity in the Galaxy along the line of sight to the HDFN. Introducing the effects of dust absorption on model fluxes in the observed frame following the Milky Way extinction curve (Seaton 1979) with $E(B-V)=0.012$, as inferred from the maps of Schlegel et al. (1998), we obtain minor changes in the redshift estimates, though in the direction of improving the agreement with spectroscopic redshifts. Using the BC code, for example, we find $\sigma_{z}=0.071, \overline{\Delta z}=0.002$ at $z_{\text {spec }}<1.5$, and $\sigma_{z}=0.117, \overline{\Delta z}=0.036$ at $z_{\mathrm{spec}} \geq 2.0$.

Dawson et al. (2001) recently published 12 new redshifts within the HDFN. Adding this subset to our sample (but excluding galaxies 2-600.0 and 4-236.0, which have a very tentative redshift), we obtain $\sigma_{z} /\left(1+z_{\text {spec }}\right) \sim 0.040$ on the largest deep spectroscopic sample available up to date (155 objects). This is a factor of 2.0-2.5 better than previous estimates in the literature. We find $\sigma_{z}=0.070$, 


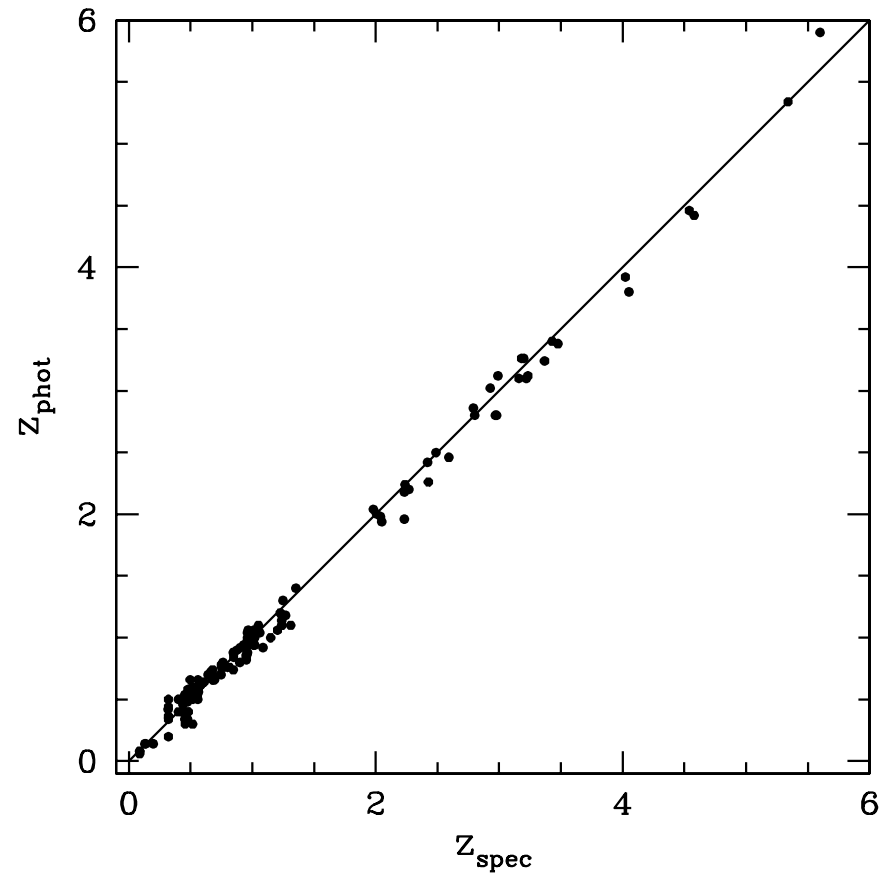

Fig. 7. Comparison between spectroscopic redshifts and photometric estimates obtained with the BUZ library plus Leitherer et al. (1999) models in the entire redshift interval $z \in[0,6]$. At $z_{\text {spec }} \geq 2$, the ISM and IGM opacities follow the rules introduced in this work. The solid line is for $\Delta z=0.0$.

$\overline{\Delta z}=0.001$ at $z_{\text {spec }}<1.5$, and $\sigma_{z}=0.116, \overline{\Delta z}=0.034$ at $z_{\text {spec }} \geq 2.0$. Figure 8 shows the distribution of residuals between $z_{\text {spec }}$ and $z_{\text {phot }}$, normalized to $\left(1+z_{\text {spec }}\right)$. It suggests that all estimated redshifts are confined in the range $-0.1 \leq\left(z_{\text {spec }}-z_{\text {phot }}\right) /\left(1+z_{\text {spec }}\right) \leq 0.1$, confirming the reliability of the SED fitting procedure.

\subsection{The faint limit}

Our results state the accuracy of the SED fitting method when applied to the bright spectroscopic sample. When galaxies are fainter and photometric errors increase the accuracy obviously decreases.

We used a simple Monte Carlo method to separate the effects of observational uncertainties from the effects due to model incompleteness ("template mismatch") in defining the distribution of residuals between photometric and spectroscopic redshifts. We generated 100 copies of the spectroscopic sample, adding to each galaxy flux a correction randomly extracted from the Gaussian distribution of its photometric error.

We compared, for each object, the redshift obtained from the measured photometry $z_{\text {phot }}$ and that from the bootstrapped galaxy photometry $z_{\operatorname{sim}}$. Due to photometric errors, the dispersion value is $\sigma_{z}^{\text {err }} /\left(1+z_{\text {phot }}\right) \sim 0.031$, and the systematic error goes to zero.

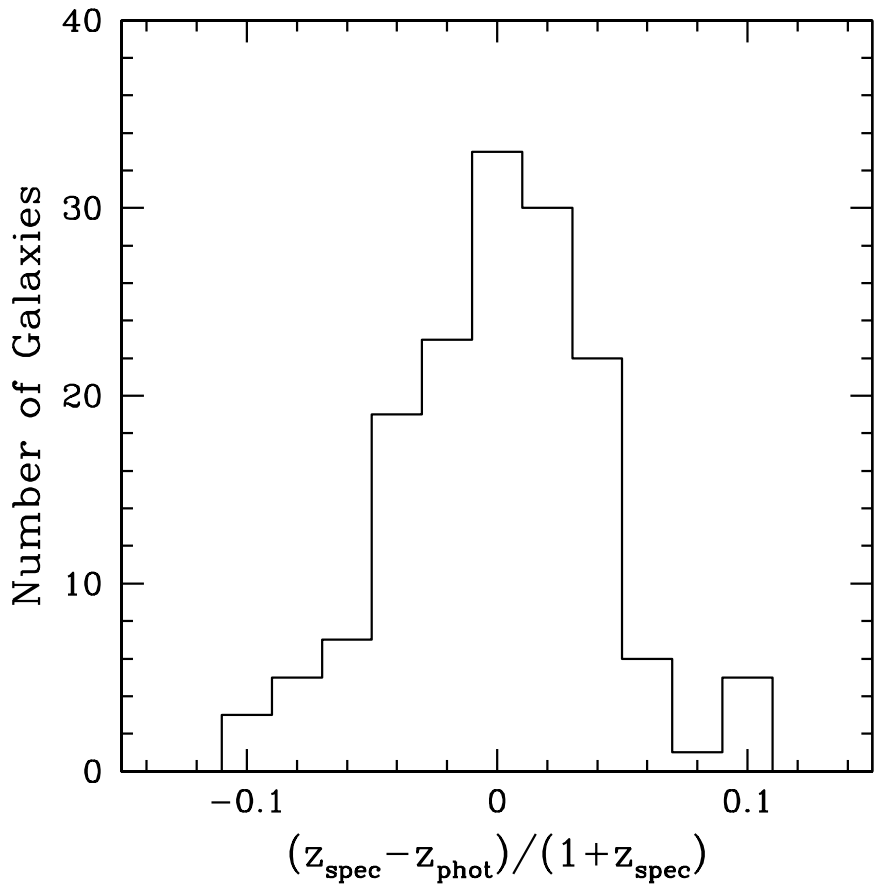

Fig. 8. The distribution of residuals between $z_{\mathrm{spec}}$ and $z_{\text {phot }}$, normalized to $\left(1+z_{\text {spec }}\right)$.

To quantify the contribution of model incompleteness (and only that) to the dispersion, we assume that the two sources of error are uncorrelated. In this case,

$\sigma_{z}^{2} \sim\left(\sigma_{z}^{\mathrm{err}}\right)^{2}+\left(\sigma_{z}^{\mathrm{temp}}\right)^{2}$

and we obtain $\sigma_{z}^{\text {temp }} /\left(1+z_{\text {spec }}\right) \sim 0.025$.

Since the residuals introduced by template incompleteness are negligible compared to the photometric redshift instability caused by observational uncertainties in the intermediate and faint galaxy sample (see Paper I, Table 2), we can conclude that, at the faint limit, Monte Carlo methods are sufficient to estimate errors in the SED technique.

In Fig. 9 we show the redshift distribution of HDFN galaxies obtained by applying our photometric redshift code to the entire catalog of Fernández-Soto et al. (1999). The well known peak at $z \sim 0.9$ is clearly visible and there is a gradual decline at larger redshifts, with a tail at very high redshift $z>4.5$.

\section{Discussion and conclusions}

We have explored the accuracy of the SED fitting method to estimate photometric redshifts by comparing its results to spectroscopic measures from the deepest and most complete redshift catalog to date in the HDFN (Cohen et al. 2000) complemented by new HDF South redshift data. We used as photometric dataset the Fernández-Soto et al. (1999) catalog for the HDF North, and for the HDF South the catalogue from the Stony Brook's group (web page http: //www.ess.sunysb.edu/astro/hdfs). The libraries of galaxy colors were supplied by the theoretical 


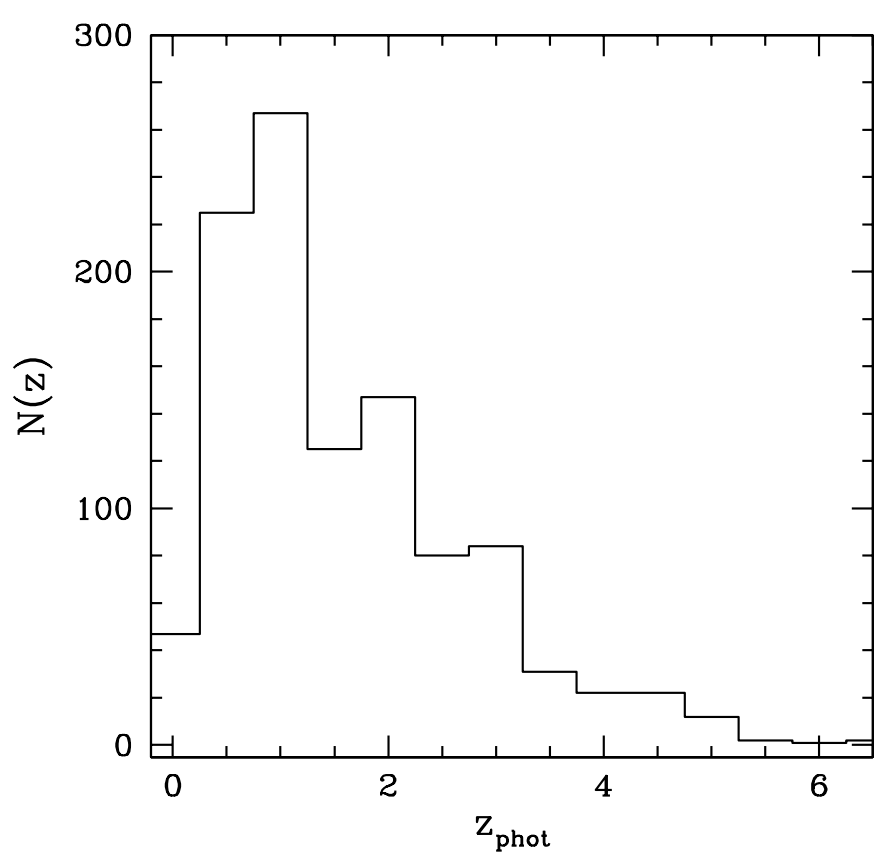

Fig. 9. The redshift distribution of HDFN galaxies.

codes of Buzzoni (2001), Bruzual \& Charlot (1993), and Fioc \& Rocca-Volmerange (1997) to reproduce normal galaxy SEDs for different morphological types, and by the theoretical code by Leitherer et al. (1999) to describe starburst SEDs.

A very good agreement between spectroscopic and photometric redshifts is found at $z_{\text {spec }}<1.5$, independently of the template set used. Model libraries include enough information to properly reproduce galaxy SED as a function of redshift from the ultraviolet to nearinfrared. At $z_{\text {spec }} \geq 2$ the usual recipes for quantifying ISM and IGM absorption yield photometric redshift estimates that are systematically smaller than the spectroscopic ones $(\overline{\Delta z}=0.156)$, while the dispersion increases to $\sigma_{z}=0.177$, confirming previous studies.

We focussed our attention on the prescriptions used to describe photon absorption in the IGM and ISM, since these processes have been shown (Paper I) to be the deciding factors in the redshift estimate at $z_{\text {spec }} \geq 2$.

In the redshift range $z \in[1.5,3]$ the Madau (1995) prescription (adopting the Press et al. 1993 values) overestimates IGM opacities with respect to the values obtained by Scott et al. (2000) on a larger quasar sample. The observed deficit in quasar fluxes due to the cumulative absorption by the Ly $\alpha$ lines of the IGM cannot be described with a single power law because the slope of $D_{\mathrm{A}}(z)$ changes in the interval $z \in[3,4]$. We adopted the Scott et al. (2000) values at $z<3$ and Press et al. (1993) values at $z>4$, and a linear interpolation between these two estimates in the range $z \in[3,4]$. In this way at $z>2.5$ the tendency to underestimate galaxy redshifts disappears and at the same time the dispersion decreases $\left(\sigma_{z}=0.129\right)$.
In the range $z_{\text {spec }} \in[2,2.5], 3$ objects in the spectroscopic sample show optical colors $\left(B_{450}-V_{606}<V_{606}-\right.$ $\left.I_{814}\right)$ incompatible with intrinsic model colors for starburst galaxies and with the dust reddening absorption law of Calzetti (1999). The common feature is a flux dimming in the $V_{606}$ band, which samples the restframe wavelength region $\lambda \sim 2000 \pm 300 \AA$ at $z \sim 2$. Adding to the dust attenuation law a weak $(\alpha=0.1,0.25) 2175 \AA$ bump, enables template colors to reproduce the observed colors of high redshift starburst galaxies in the $\left(B_{450}-V_{606}\right)$ vs. $\left(V_{606}-I_{814}\right)$ plane.

When these improvements are implemented, the dispersion between photometric and spectroscopic redshifts at $z_{\text {spec }} \geq 2.0$ becomes $\sigma_{\mathrm{z}}=0.116$, and the systematic error $\overline{\Delta z}=0.034$. In the entire redshift interval $z \in[0,6]$, we obtain the value $\sigma_{\mathrm{z}} /\left(1+z_{\text {spec }}\right) \sim 0.04$. This is an improvement by a factor $\sim 2.0-2.5$ over previous estimates in the literature.

Acknowledgements. We would like to thank the referee A. Fernández-Soto for his careful reading of the manuscript and useful comments. This work received partial financial support by Fondazione Cariplo and COFIN grant 00-02-016. A.I. and D.V.-G. thank ESO for financial support during a period spent at ESO-Santiago, where part of this work was done.

\section{References}

Arnouts, S., Cristiani, S., Moscardini, L., et al. 1999, MNRAS, 310,540

Bolzonella, M., Miralles, J. M., \& Pelló, R. 2000, A\&A, 363, 476

Bruzual, G. A., \& Charlot, S. 1993, ApJ, 405, 538

Buzzoni, A. 1989, ApJS, 71, 817

Buzzoni, A. 1995, ApJS, 98, 69

Buzzoni, A. 2001, AJ, submitted

Calzetti, D. 1999, Ap\&SS, 266, 243

Calzetti, D. 2000, FIRSED2000 Workshop on The Far-Infrared and Submillimiter Spectral Energy Distributions of Active and Starburst Galaxies, ed. P. Barthel, B. Wilkes, \& I. van Bemmel, New Astr. Rev. (Elsevier, The Netherlands) [astro-ph/0008403]

Calzetti, D., Kinney, A., \& Storchi-Bergmann, T. 1994, ApJ, 429,582

Cohen, J. G., Hogg, D. W., Blandford, R., et al. 2000, ApJ, 538,29

Cohen, J. G. 2001, AJ, 121, 2895

Connolly, A. J., Szalay, A. S., Dickinson, M., et al. 1997, ApJ, 486, L11

Connolly, A. J., Szalay, A. S., \& Brunner, R. J. 1998, ApJ, 499, L125

Cristiani, S., Appenzeller, I., Arnouts, S., et al. 2000, A\&A, 359,489

Da Costa, L., Nonino, M., Rengelink, R., et al. 2001, in preparation [astro-ph/9812105]

Dawson, S., Stern, D., Bunker, A. J., et al. 2001, AJ, 122, 598

Dickinson, M. 1998, in The Hubble Deep Field, ed. M. Livio, S. M. Fall, \& P. Madau (Cambridge Univ. Press, New York), 219 (see also http://www.stsci.edu/ftp/ science/hdf/clearinghouse/irim/irim_hdf.html)

Fan, X., White, R. L., Davis, M., et al. 2000, AJ, 120, 1167 
Ferguson, H., Dickinson, M., \& Williams, R. E. 2000, ARA\&A, Meurer, G. R., Heckman, T. M., Lehnert, M. D., et al. 1997, 38,667 AJ, 114, 54

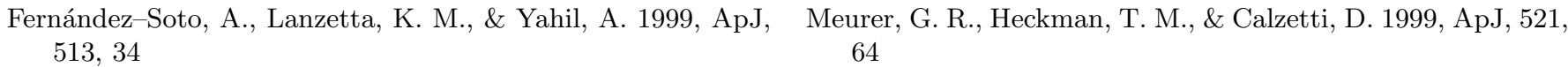

Fernández-Soto, A., Lanzetta, K. M., Chen, Hsiao-Wen, et al. Miralles, J. M., \& Pelló, R. 1998, ApJ, submitted 2001, ApJS, 135, 41

Fitzpatrick, E. L. 1986, AJ, 92, 1068

Fitzpatrick, E. L., \& Massa, D. 1988, ApJ, 328, 734

Fontana, A., D'Odorico, S., Poli, F., et al. 2000, AJ, 120, 2206

Fioc, M., \& Rocca-Volmerange, B. 1997, A\&A, 326, 950

Franceschini, A., Silva, L., Fasano, G., et al. 1998, ApJ, 506, 600

Glazebrook, K., et al. 2001, in preparation (see also http:// www . aao.gov . au/hdfs)

Gordon, K. D., Calzetti, D., \& Witt, A. N. 1997, ApJ, 487, 625

Granato, G. L., Lacey, C. G., Silva, L., et al. 2000, ApJ, 542, 710

Leitherer, C., Schaerer, D., Goldader, J. D., et al. 1999, ApJS, 123,3 [astro-ph/9801062]

Pascarelle, S. M., Lanzetta, K. M., \& Fernández-Soto, A. 1998, ApJ, 508, L1

Press, W. H., Rybicki, G. B., \& Schneider, D. P. 1993, ApJ, 414, 64

Rodighiero, G., Franceschini, A., \& Fasano, G. 2001, MNRAS, 324,491

Roukema, B. F., Valls-Gabaud, D., Mobasher, B., \& Bajtlik, S. 1999, MNRAS, 305, 151

Sawicki, M. J., Lin, H., \& Yee, H. K. C. 1997, AJ, 113, 1

Sawicki, M. J., \& Yee, H. K. C. 1998, AJ, 115, 1418

Schlegel, D. J., Finkbeiner, D. P., \& Davis, M. 1998, ApJ, 500, 525

Scott, J., Bechtold, J., \& Dobrzycki, A. 2000, ApJS, 130, 37

Lowenthal, J. D., Koo, D. C., Guzman, R., et al. 1997, ApJ, 481, L673

Madau, P. 1995, ApJ, 441, 18

Madau, P., Ferguson, H. C., Dickinson, M., et al. 1996, MNRAS, 283, 1388

Massarotti, M., Iovino, A., \& Buzzoni, A. 2001a, A\&A, 368, 74 (Paper I)

Massarotti, M., Iovino, A., \& Buzzoni, A. 2001b, ApJL, in press [astro-ph/0109013]

Seaton, M. J. 1979, MNRAS, 187, 73

Silva, L., Granato, G. L., Bressan, A., et al. 1998, ApJ, 509, 103

Spinrad, H., Stern, D., Bunker, A., et al. 1998, AJ, 116, 2617

Williams, R. E., Blacker, B., Dickinson, M., et al. 1996, AJ, 112,1335

Zuo, L., \& Lu, L. 1993, ApJ, 418, 601 